\title{
Triplicate PCR reactions for 16S rRNA gene amplicon sequencing are unnecessary
}

Clarisse Marotz' ${ }^{1}$ Anukriti Sharma ${ }^{2}$, Greg Humphrey' ${ }^{\prime}$ Neil Gottel $^{2}$, Christopher Daum ${ }^{3}$, Jack A Gilbert ${ }^{2}$, Emiley Eloe-Fadrosh ${ }^{3}$ \& Rob Knight*,1,45

\begin{abstract}
Conventional wisdom holds that PCR amplification for sequencing should employ pooled replicate reactions to reduce bias due to jackpot effects and chimera formation. However, modern amplicon data analysis employs methods that may be less sensitive to such artifacts. Here we directly compare results from single versus triplicate reactions for $16 \mathrm{~S}$ amplicon sequencing and find no significant impact of adopting a less laborintensive single reaction protocol.
\end{abstract}

\section{METHOD SUMMARY}

We compared single PCR reactions to pooled triplicate $\mathrm{PCR}$ reactions for 16S rRNA gene amplicon sequencing on nearly 400 samples from a diverse range of environments across three independent laboratories.

\section{KEYWORDS}

16S rRNA gene amplicon sequencing - microbiome $\cdot \mathrm{PCR} \cdot$ replicate PCR reactions

Department of Pediatrics, University of California, San Diego, La Jolla, CA, USA; ${ }^{2}$ Division of Bioscience, Argonne National Laboratory University of Chicago, Chicago, IL, USA; ${ }^{3}$ Department of Energy Joint Genome Institute, Walnut Creek, CA, USA: ${ }^{4}$ Center for Microbiome Innovation, University of California San Diego, La Jolla, CA, USA; ${ }^{5}$ Department of Computer Science and Engineering, University of California San Diego, La Jolla, CA, USA; *Author for correspondence: robknight@ucsd.edu

BioTechniques 67: 29-32 (July 2019) 10.2144/ btn-2018-0192
For decades, 16S rRNA gene sequencing has been performed by pooling replicate PCR reactions, usually in triplicate. The primary benefit is to reduce 'jackpotting': the stochastic nature of PCR means that some molecules are amplified earlier than others, and exponential amplification in subsequent rounds of PCR substantially distort the frequencies of different molecules in heterogeneous pools of target genes [1]. This phenomenon is particularly important in environmental DNA sequencing where the goal is an accurate, or at least consistent, readout of the different gene targets matching a primer set.

However, since the guideline that PCR should be performed in triplicate was introduced [1], there have been substantial improvements in the processivity and fidelity of DNA polymerases. Therefore, triplicate PCR may no longer provide the benefits it once did, although performing single PCR reactions instead of triplicate would provide significant time and cost savings. Several studies have tested single versus triplicate PCR for 16S rRNA sequencing in limited settings with a small number of input samples (e.g., 18 soil samples [2], two soil and two stool samples [3], three soil samples [4]). However, it has never been tested across the wide range of samples and settings that would be needed to justify a general recommendation for change in protocol. We used the availability of standardized sample sets such as those from MBQC, the Microbiome Quality Control project [5], and from our previous technology testing to answer this question definitively across three different laboratories. In total, we tested the effects of replicate $\mathrm{PCR}$ pooling in three independent experiments containing nearly 373 samples from a diverse range of environments.

First, we benchmarked single versus pooled-triplicate PCR across a broad range of sample types. In our previous study on comparison of DNA extraction methods [6] we assembled a set of 96 samples spanning a broad range of environments, including 48 fecal samples, 12 soil samples, 12 marine sediment samples, six seawater samples, five skin samples, five oral samples, and six mattress dust samples. We used the DNA from this previous study, extracted using the Earth Microbiome Project protocol [7] on the Kingfisher instrument, for this study. 16S rRNA gene amplification was performed according to the Earth Microbiome Project (EMP) protocol and is detailed in the supplemental file. We quantified amplicons by PicoGreen $^{\text {TM }}$ and pooled $240 \mathrm{ng}$ of each for sequencing. We ran the entire sample set four times: twice with single PCR and twice with pooled-triplicate PCR. The pooled library was sequenced on the Illumina MiSeq sequencing platform with a MiSeq Reagent Kit v2 and paired-end 150 cycles. All data were processed and analyzed using the QIIME2 software suite [8] and Deblur [9]. Counterintuitively, single PCR reactions yielded significantly more reads than triplicate $\mathrm{PCR}$ reactions (mean \pm SEM: $10,821 \pm 298$ versus $10,029 \pm 262$, respectively, paired T-test $p=0.0003$ ), and fewer dropouts (Figure 1A). We saw no significant difference in alpha diversity, regardless of environment (Figure 1B). Beta diversity analysis with Unweighted UniFrac demonstrates that samples cluster by sample type and not number of PCR reactions (Figure 1C). The Weighted UniFrac distances are significantly larger among samples from different environments than among biological replicates, and distances among biological replicates are significantly greater than technical replicates, with both single and triplicate PCR reactions (Figure 1D). Negligible taxonomic changes between single and triplicate reactions were observed $(97.8 \%$ shared taxonomy at the species level, genus $98.4 \%$, and phylum $100 \%$, Supplementary Figure 1A \& Supplementary Figure 2).

Second, because high-level conclusions crossing environment types might obscure relationships in particular sample types, we tested whether the conclusions held for a separate set of agricultural 


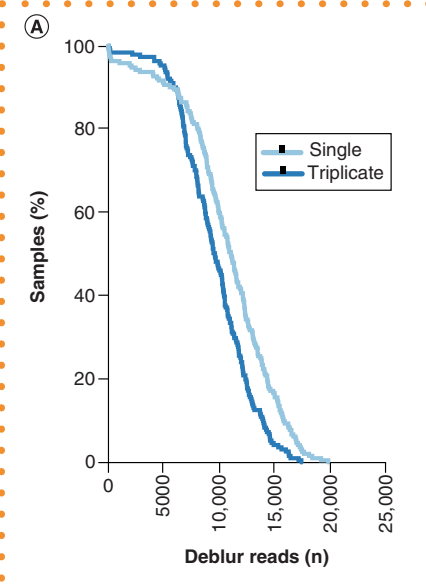

(B)

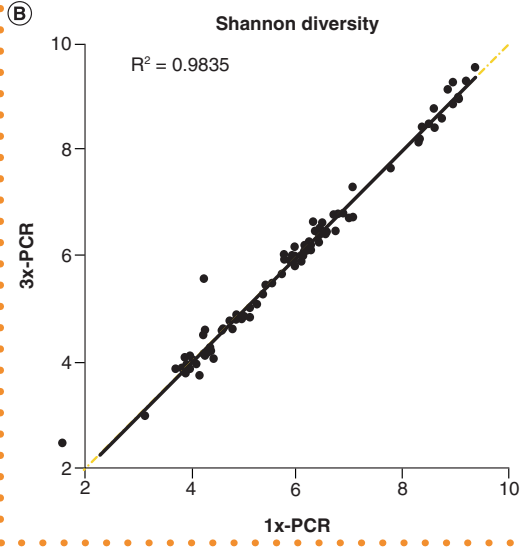

(C)

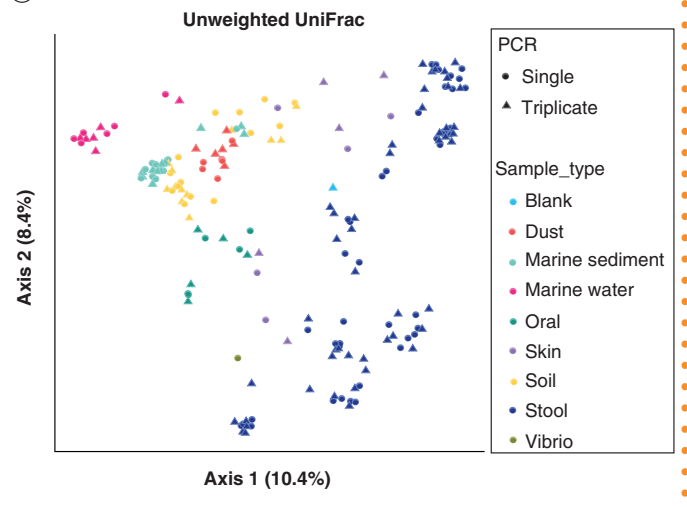

samples does not differ in single versus triplicate PCRs (Figure 2C \& D). Negligible taxonomic changes between single and triplicate reactions were observed (99.3\% shared taxonomy at the species level, genus $99.2 \%$, and phylum 100\%, Supplementary Figure 1B \& Supplementary Figure 3).

Finally, the microbiology of the built environment has been a rapidly expanding topic of interest over the past decade but poses unique challenges for molecular analysis. In particular, samples tend to be contaminated with high levels of human DNA and have low bacterial biomass [10]. We used samples from a previous study that collected 96 samples longitudinally from four commonly used building materials maintained at a high relative humidity ( 94\%) [11]. Genomic DNA was extracted from environmental samples using the PowerSoil DNA isolation kit as previously described [12], and genomic DNA was amplified using the EMP protocol as detailed in the supplemental file. Samples were processed both with single PCR and pooledtriplicate $\mathrm{PCR}$ reactions, and sequenced on an Illumina MiSeq sequencing platform with a MiSeq Reagent Kit v2 and paired-end 150 cycles. Once again, yields were higher with single PCR than triplicate PCR (Figure 3A), Shannon diversity was not affected by single versus triplicate PCR (Figure 3B), and beta diversity was driven by biological parameters of the sample rather than by single versus triplicate PCR (Figure $3 C$ \& D). Negligible taxonomic changes between single and triplicate reactions were observed (96.5\% shared taxonomy at the species level, genus $95.8 \%$, and phylum $100 \%$, Supplementary Figure 1C \& Supplementary Figure 4). All data from each of the three experiments are publicly available from the EBI under accession number ERP113817.

Taken together, these results demonstrate that with modern methods pooling triplicate PCR reactions for $16 \mathrm{~S}$ rRNA amplicon sequencing is more expensive and does not provide improvement over single PCR reactions. This result was confirmed in studies spanning three laboratories, hundreds of samples, and numerous distinct environment types. However, although these results hold true for the range of conditions tested here, there are so many variations in PCR techniques that this type of benchmarking effort should be 
validated for specific sample types and PCR protocols before a switch from established procedure is implemented for specialized protocols. For the general sample types tested here, we recommend using single PCRs rather than triplicate PCRs. Combined with other technical improvements in miniaturizing PCR reactions [13], this change in protocol will substantially reduce the cost and complexity of amplicon studies.

\section{AUTHOR CONTRIBUTIONS}

$\mathrm{RK}, \mathrm{JG}$, and EE designed the experiments, $\mathrm{GH}, \mathrm{NG}$, and $\mathrm{CD}$ ran the experiments, $\mathrm{CM}$ and AS analyzed the data, and RK and CM wrote the manuscript.

\section{FINANCIAL \& COMPETING INTERESTS DISCLOSURE}

Data from JGI was collected with support from a grant from the Office of Science (BER), US Department of Energy, Grant no DE-SC0014395 to Daniel Schachtman and Susannah Tringe. The work conducted by the US Department of Energy Joint Genome Institute, a DOE Office of Science User Facility, is supported under Contract No. DE-AC02-05CH11231. Clarisse Marotz was supported by NIDCR F31 Fellowship 1F31DE028478. The authors have no other relevant affiliations or financial involvement with any organization or entity with a financial interest in or financial conflict with the subject matter or materials discussed in the manuscript apart from those disclosed.

No writing assistance was utilized in the production of this manuscript.

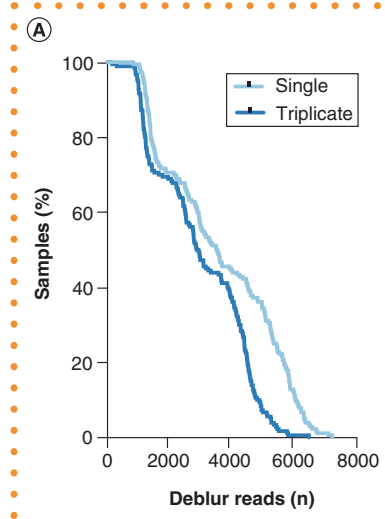

(B)

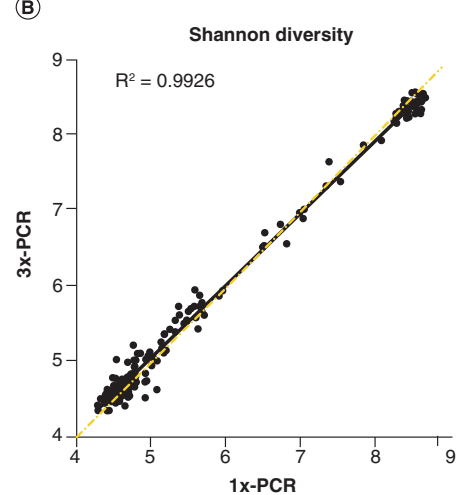

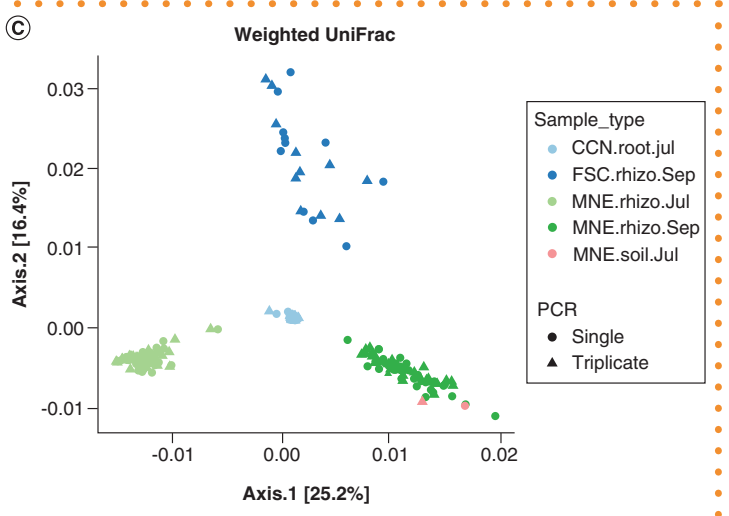

(D)

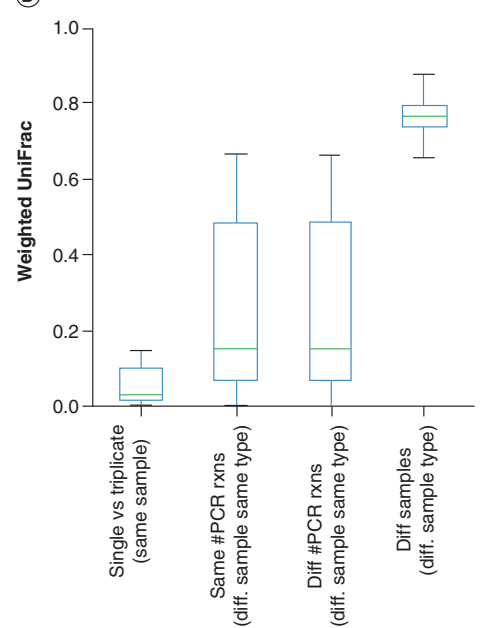

Figure 2. Effect of 16S PCR reaction number across agricultural samples. (A). The sequencing dropout number of all samples run with either single or triplicate PCR reactions. (B) Shannon diversity index of each sample is similar between single and triplicate PCR reactions. (C) Weighted UniFrac PCoA plot shows that samples cluster by sample type (color) and not number of PCR reactions (shape). (D) Weighted Unifrac distances between single or triplicate PCR reactions of the same sample are smaller than the distance between different samples of the same type run with either single or triplicate PCR reactions, and both are smaller than the distance between samples from different environments.

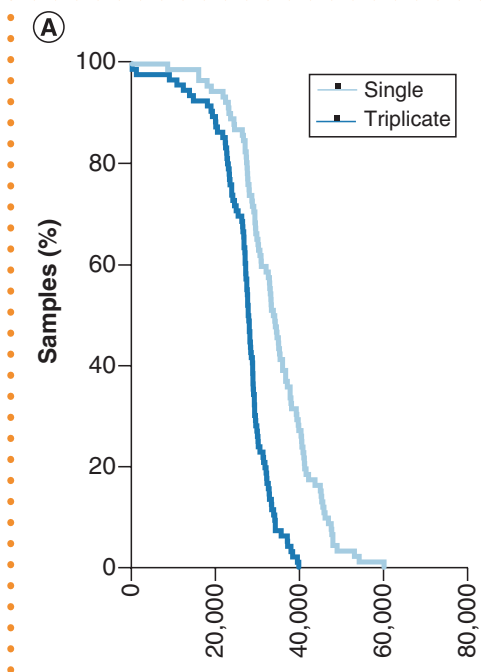

Normalized deblur reads $(\mathrm{n})$

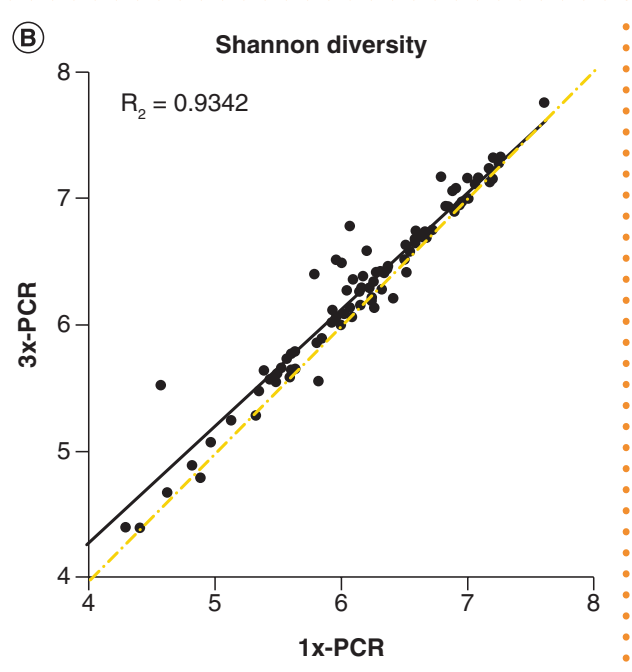

Figure 3. Effect of 16S PCR reaction number across building materials. (A) The sequencing dropout number of all samples run with either single or triplicate PCR reactions. (B) Shannon diversity index is similar between single and triplicate PCR reactions. (C) Weighted UniFrac PCoA plot shows that samples do not cluster by number of PCR reactions (shape). (D) Weighted UniFrac distances between single or triplicate PCR reactions of the same sample are smaller than the distance between different samples with either single or triplicate PCR reactions. 
:C)

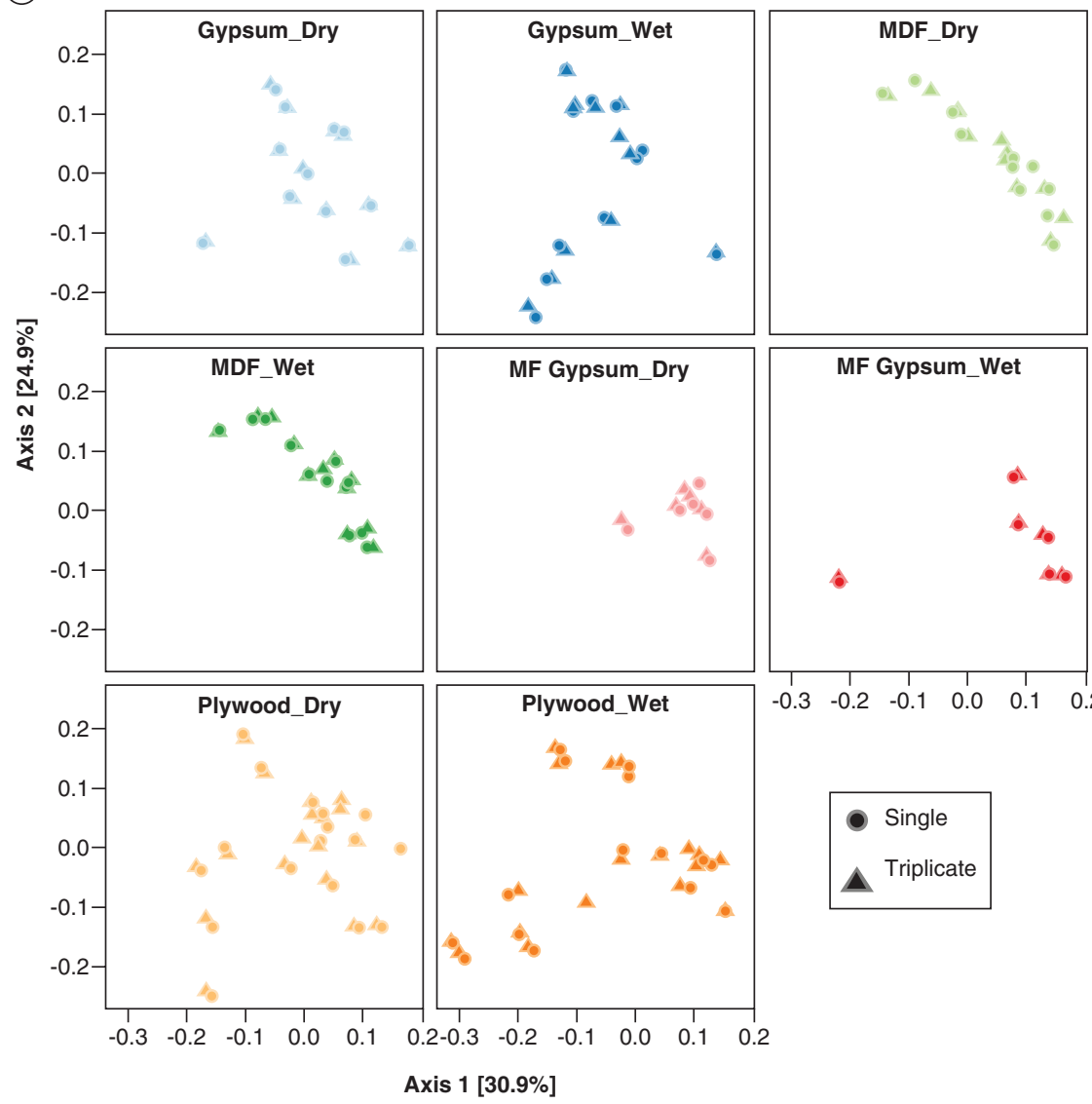

(D)

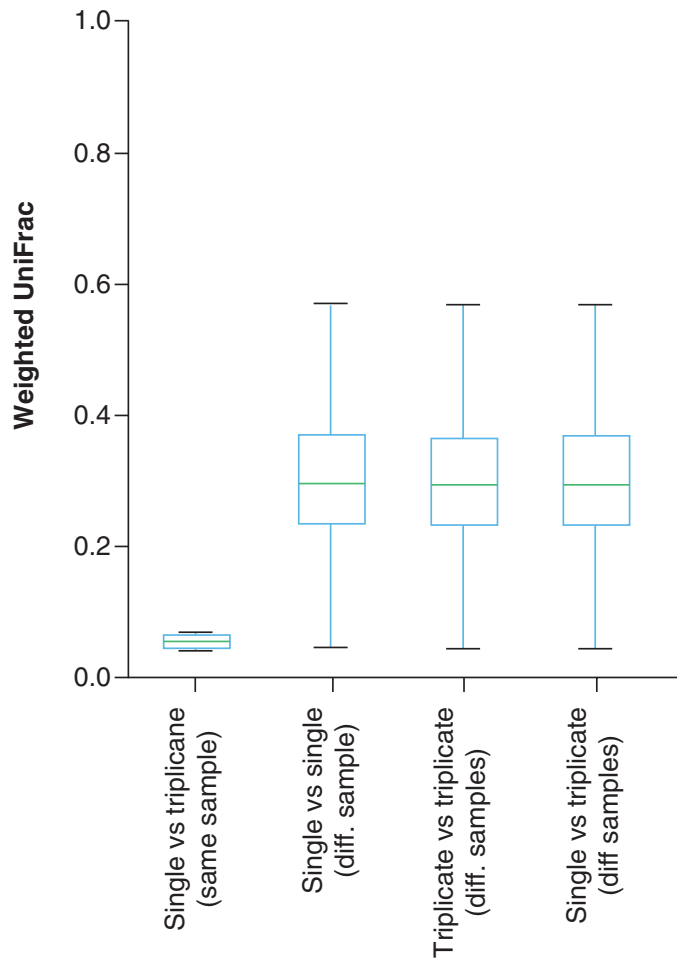

Figure 3 (cont.). Effect of 16S PCR reaction number across building materials. (A) The sequencing dropout number of all samples run with either single or triplicate PCR reactions. (B) Shannon diversity index is similar between single and triplicate PCR reactions. (C) Weighted UniFrac PCoA plot shows that samples do not cluster by number of PCR reactions (shape). (D) Weighted UniFrac distances between single or triplicate PCR reactions of the same sample are smaller than the distance between different samples with either single or triplicate $\mathrm{PCR}$ reactions.

\section{$\checkmark$ OPEN ACCESS}

This work is licensed under the AttributionNonCommercial-NoDerivatives 4.0 Unported License. To view a copy of this license, visit http://creativecommons.org/licenses/ by-nc-nd/4.0/

\section{SUPPLEMENTARY DATA}

To view the supplementary data that accompany this paper please visit the journal website at: www.future-science. com/doi/suppl/10.2144/btn-2018-0192

\section{REFERENCES}

1. Polz MF, Cavanaugh CM. Bias in template-to-product ratios in multitemplate $P C R$. Appl. Environ Microbiol. 64(10), 3724-3730 (1998).

2. Wen $\mathrm{C}, \mathrm{Wu} \mathrm{L}$, Qin $\mathrm{Y}$ et al. Evaluation of the reproducibility of amplicon sequencing with Illumina MiSeq platform. PLoS One 12(4), e0176716 (2017).

3. Kennedy K, Hall MW, Lynch MDJ, Moreno-Hagelsieb G Neufeld JD. Evaluating bias of illumina-based bacterial 16S rRNA gene profiles. 80(18), 5717-5722 (2014).

4. Smith DP, Peay KG. Sequence depth, not PCR replication, improves ecological inference from next generation DNA sequencing. PLoS One 9(2), e90234 (2014).

5. Sinha R, Abu-Ali G, Vogtmann E et al. Assessment of variation in microbial community amplicon sequencin by the microbiome quality control (MBQC) project consortium. Nat. Biotechnol. 35(11), 1077 (2017).

6. Marotz C, Amir A, Humphrey G, Gaffney J, Gogul G, Knight R. DNA extraction for streamlined metagenomics of diverse environmental samples. Biotechniques 62(6), 290-293 (2017).

7. Gilbert J, Jansson J, Knight R. The earth microbiome project. (2010). www.earthmicrobiome.org

8. Bolyen E, Rideout JR, Dillon MR et al. QIIME 2: reproducible, interactive, scalable, and extensible microbiome data science. No. e27295v1. Peer J. Preprints (2018).

9. Amir A, McDonald D, Navas-Molina JA et al. deblur rapidly resolves single-nucleotide community sequence patterns. mSystems 2(2), e00191-16 (2017).

10. Kelley ST, Gilbert JA. Studying the microbiology of the indoor environment. Genome Biol. 14(2), 202 (2013).

11. Cardona C, Lax S, Larsen P et al. Environmental sources of bacteria differentially influence host-associated microbial dynamics. MSystems 3(3), e00052-18 (2018).

12. McPherson MR, Wang $P$, Marsh EL, Mitchell RB, Schachtman DP. Isolation and analysis of microbial communities in soil, rhizosphere, and roots in perennia grass experiments. J. Vis. Exp. (137), e57932 (2018).

13. Minich JJ, Humphrey G, Benitez RAS et al. High-throughput miniaturized 16S rRNA amplicon library preparation reduces costs while preserving microbiome integrity. 